Case Report

\title{
Chronic Myeloid Leukemia with an e6a2 BCR-ABL1 Fusion Transcript: Cooperating Mutations at Blast Crisis and Molecular Monitoring
}

\author{
Mireille Crampe, ${ }^{1}$ Karl Haslam, ${ }^{1}$ Emma Groarke, ${ }^{2}$ Eileen Kelleher, ${ }^{3}$ Derville O'Shea, \\ Eibhlin Conneally, ${ }^{2}$ and Stephen E. Langabeer ${ }^{1}$
}

${ }^{1}$ Cancer Molecular Diagnostics, St. James's Hospital, Dublin 8, Ireland

${ }^{2}$ Department of Haematology, St. James's Hospital, Dublin 8, Ireland

${ }^{3}$ Department of Haematology, Bon Secours Hospital, Cork, Ireland

${ }^{4}$ Department of Haematology, Cork University Hospital, Cork, Ireland

Correspondence should be addressed to Stephen E. Langabeer; slangabeer@stjames.ie

Received 10 July 2017; Revised 13 September 2017; Accepted 17 September 2017; Published 16 October 2017

Academic Editor: Sergio Storti

Copyright (C) 2017 Mireille Crampe et al. This is an open access article distributed under the Creative Commons Attribution License, which permits unrestricted use, distribution, and reproduction in any medium, provided the original work is properly cited.

\begin{abstract}
A minority of chronic myeloid leukemia patients (CML) express a variety of atypical $B C R-A B L 1$ fusion variants and, of these, the e6a2 BCR-ABL1 fusion is generally associated with an aggressive disease course. Progression of CML to blast crisis is associated with acquisition of additional somatic mutations yet these events have not been elucidated in patients with the e6a2 $B C R-A B L 1$ genotype. Moreover, molecular monitoring is only sporadically performed in CML patients with atypical $B C R-A B L 1$ fusion transcripts due to lack of consensus approaches or standardization. A case of CML is described in which comprehensive molecular analysis, including targeted next-generation sequencing, revealed a single $A S X L 1$ mutation cooperating with an e6a2 BCR-ABL1 fusion transcript at blast crisis. A quantitative molecular monitoring approach was devised and adopted that reflected the disease response from initial treatment through allogeneic stem cell transplantation which resulted in undetectable e6a $2 B C R-A B L 1$ transcripts. This case emphasizes the requirement for molecular monitoring in CML patients with atypical $B C R-A B L 1$ fusion transcripts and emphasizes that comprehensive sequencing has the potential to identify targets for novel therapies in CML patients with advanced disease.
\end{abstract}

\section{Introduction}

The molecular genetic hallmark of chronic myeloid leukemia (CML) is the $B C R-A B L 1$ oncogene resulting from the $\mathrm{t}(9 ; 22)$ translocation which fuses $A B L 1$ on chromosome $9 \mathrm{q} 34$ to $B C R$ on chromosome 22q11. The vast majority of CML patients possess either e13a2 or e14a2 BCR-ABL1 fusion transcripts; however, several alternative transcripts have been reported that are largely the result of either $B C R$ or $A B L 1$ alternative exon splicing. These uncommon, variant transcripts can result in phenotypic variability and affect response to tyrosine kinase inhibitor (TKI) therapy [1]. Since the initial molecular characterization of the e6a $2 B C R-A B L 1$ transcript that fuses $B C R$ exon 6 to $A B L 1$ exon a2 [2], less than twenty CML cases have been reported in which the e6a2 fusion is usually associated with a clinically aggressive disease frequently presenting in accelerated or blast crisis phases [3-6]. This transcript type has also been reported in $t(9 ; 22)$-positive acute myeloid leukemia (AML) and transformed chronic myelomonocytic leukemia [7-9]. Although responses to imatinib have been reported [10,11], several cases of $A B L 1$ kinase domain mutation-associated imatinib resistant, e6a2 $B C R-A B L 1$ CML have been documented $[12,13]$ with limited information on the efficacy of front line second-generation TKIs in this genotype. As e6a $2 B C R-A B L 1$ CML is associated with aggressive disease, allogeneic stem cell transplantation (ASCT) remains the only curative option and in such circumstances conventional AML therapy has been combined with a second-generation TKI as a bridge to ASCT [14-16]. 


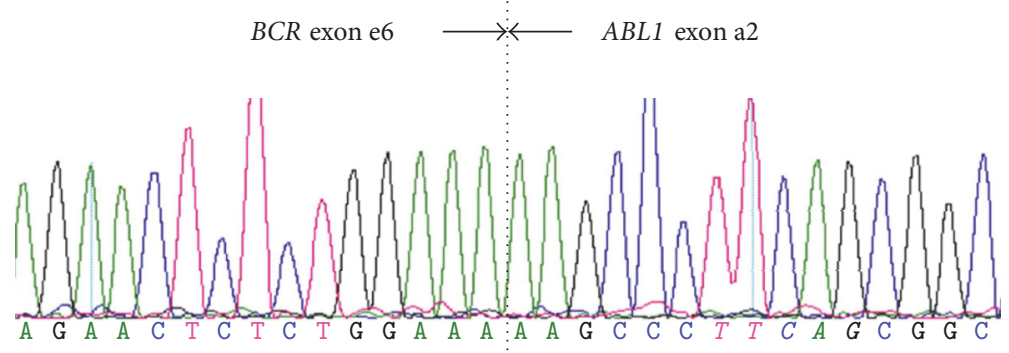

FIgURE 1: Sanger sequencing of $B C R-A B L 1$ breakpoint demonstrating fusion of $B C R$ exon 6 to $A B L 1$ exon a2.

Acquisition of further cytogenetic abnormalities and AML-associated, disease driving mutations are common findings in blast crisis of CML [17] but whether these additional events impact on prognosis is unclear [18]. Analogous to AML, identification of cooperating mutations may establish targets for novel therapeutic interventions in addition to TKI therapy [19]. Information on the incidence and pattern of these cooperating mutations in CML patients with the rare fusion transcript types is lacking.

Molecular monitoring of $B C R-A B L 1$ transcripts by reverse transcription-quantitative polymerase chain reaction (RT-qPCR) is an integral part of the modern management of CML patients with achievement of molecular milestones according to internationally standardized practice incorporated into current management guidelines [20, 21]. However, standardization of these techniques applies only to the common e13a2 and e14a2 BCR-ABL1 breakpoints with quantitative $B C R-A B L 1$ levels reported on an ad hoc basis in those patients with the rare, variant $B C R-A B L 1$ fusions.

A case of e6a2 $B C R-A B L 1$ CML presenting in blast crisis is reported in which a targeted next-generation sequencing (NGS) approach was utilised to ascertain additional cooperating mutations. Furthermore, an RT-qPCR approach was developed to prospectively monitor the atypical $B C R-A B L 1$ transcripts patient throughout the disease course.

\section{Case Report}

A 48-year-old female presented with a leucoerythroblastic blood film, anemia (hemoglobin $10.9 \mathrm{~g} / \mathrm{dL}$ ), thrombocytopenia $\left(72 \times 10^{9} / \mathrm{L}\right)$, normal white cell count, and no splenomegaly. An initial bone marrow (BM) aspirate was hypocellular with increased fibrosis suspected as the underlying cause. The JAK2 V617F and CALR exon nine insertion or deletion mutations were not detected but cytogenetic analysis revealed a karyotype of 46,XX,t(9;22)(q42;q11) in 16 metaphases, trisomy 8 in a further two $\mathrm{Ph}+$ metaphases, and tetrasomy 8 in one further $\mathrm{Ph}+$ metaphase. RT-qPCR analysis with standardized primers and probes [22] did not detect $B C R-A B L 1$ transcripts but further analysis with a qualitative PCR approach [23] detected a single abnormal band which Sanger sequencing demonstrated to be a fusion of $B C R$ exon 6 to $A B L 1$ exon a2 (Figure 1). No mutations were detected in the
$A B L 1$ kinase domain. Immunophenotyping of the peripheral blood (PB) demonstrated $12 \%$ myeloblasts that were positive for CD7, CD13, CD33, CD34, CD117, and HLADR and negative for CD3, CD4, CD8, CD11c, CD14, CD15, CD19, and CD64. A repeat BM aspirate was hypercellular with $18 \%$ myeloblasts morphologically. The corresponding trephine biopsy was hypercellular and had prominent grade three fibrosis with CD34+ blasts accounting for approximately $40 \%$ of the total cellularity, consistent with a diagnosis of blast crisis CML with marrow fibrosis.

In order to quantitate e6a $2 B C R-A B L 1$ transcripts, a $B C R$ exon 6 forward primer [4] was used with reverse primer ENR561 and probe ENP541 [22]. A standard curve was established by tenfold dilutions of patient presentation PB cDNA (slope -3.538, $r^{2}$ 0.996). Control $A B L 1$ transcripts were detected as previously described [22]. At diagnosis the patients' PB BCR-ABL1/ABL1 transcript level was $78.6 \%$.

An NGS approach was employed to detect additional mutations cooperating with the e6a2 $B C R-A B L 1$ fusion in driving blast crisis in this patient. Amplicon libraries covering 30 commonly mutated genes implicated in myeloid malignancies either covering the entire coding region $(C A L R$, CEBPA, ETV6, EZH2, RUNX1, SH2B3, TET2, TP53, and ZRSR2) or mutational hotspots ( $A B L 1, A S X L 1, B R A F, C B L$, CSF3R, DNMT3A, FLT3, GATA2, IDH1, IDH2, JAK2, KIT, KRAS, MPL, NPM1, NRAS, PTPN11, SETBP1, SF3B1, SRSF2, and $U 2 A F 1$ ) were generated using $20 \mathrm{ng} B \mathrm{~B}$ genomic DNA and sequencing performed using Ion AmpliSeq ${ }^{\mathrm{TM}}$ methodology (Thermo Fisher Scientific, Paisley, UK). Calling of somatic mutations was achieved using an algorithm that excluded synonymous mutations, variants located within intronic or untranslated regions, and those present at a variant allele frequency (VAF) of $<5 \%$. A minimum target depth of coverage for variant calls was set at $500 \mathrm{x}$ as previously described [24]. A single ASXL1 p.E1102D mutation (c.3306G $>$ T; reference sequence NM_015338.5) was detected with a variant allele frequency of $45.5 \%$.

The patient received one cycle of daunorubicin and cytarabine (DA $3+10$ ) with imatinib $400 \mathrm{mg}$ daily which was escalated to $600 \mathrm{mg}$ daily achieving hematological and morphological remission and resulting in a PB BCR-ABL1/ABL1 transcript level of $0.06 \%$. She was subsequently treated with a second cycle of chemotherapy (DA $3+8$ ) which was 


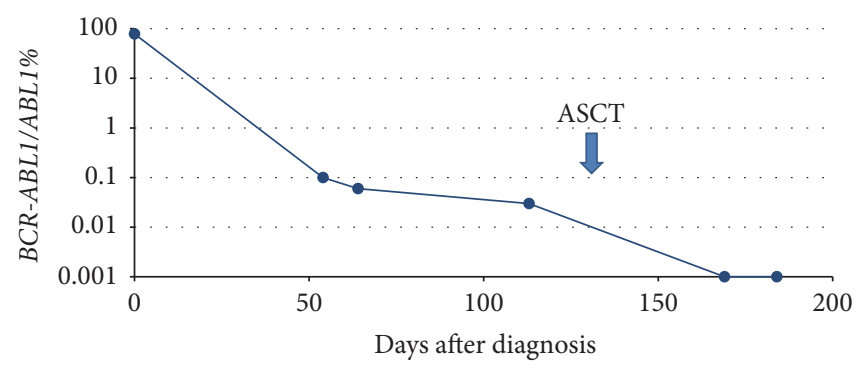

FIGURE 2: RT-qPCR of e6a2 BCR-ABL1 transcripts throughout clinical course. ASCT: allogeneic stem cell transplantation.

complicated by septicemia. Prior to ASCT the PB BCR$A B L 1 / A B L$ transcript level was $0.03 \%$ (Figure 2). Her imatinib was stopped prior to busulfan and cyclophosphamide conditioning for ASCT from a matched sibling donor that was complicated by mucositis and a coagulase-negative staphylococcus infection during her inpatient stay. After discharge she developed grade four skin graft versus host disease (GVHD) and was treated with high dose steroids subsequently developing CMV reactivation and steroid induced diabetes. Antiviral treatment with valganciclovir caused a pancytopenia requiring treatment with granulocyte colonystimulating factor. $B C R-A B L 1$ transcripts were not detected by RT-qPCR at day 38 after ASCT with full donor chimerism achieved (100\% at day 43 post-ASCT). The patient remains off TKI and generally well at last follow-up. The CMV reactivation has resolved and she continues on steroid taper for GVHD. Continued close RT-qPCR monitoring is planned.

\section{Discussion}

The e6a2 $B C R-A B L 1$ variant is rare with less than twenty CML patients of this genotype reported. Clinically, CML cases that express the variant e6a $2 B C R-A B L 1$ fusion transcript often present in advanced stages and display an aggressive disease course, confirmed by this case report: it must be noted that occasional good responses to TKI have also been documented. In the case described herein, imatinib combined with AML induction therapy resulted in a considerable reduction in the $B C R-A B L 1$ transcript level with the patient able to proceed with ASCT.

Little is known of the biological characteristics of this genetic subtype of CML and in those with other variant $B C R-A B L 1$ fusion genes. NGS targeted for prognostically and clinically relevant mutations recurrently observed in myeloid malignancies revealed a mutation of ASXL1 in this patient. ASXL1 encodes an epigenetic regulator involved in posttranslational chromatin modification with aberrant histone modification being one of the important mechanisms underlying altered epigenetic regulation in other myeloid malignancies such as the myelodysplastic syndromes (MDS) [25]. Mutations of ASXL1 and other genes involved in histone modification and DNA methylation have been characterized in chronic phase CML patients but appear to be more frequent in accelerated or blast crisis phases [26-28]. Novel therapies currently under investigation, which specifically target aberrant DNA methylation and chromatin remodelling in MDS [29], are potentially applicable to other ASXL1mutated malignancies such as blast crisis CML, suggesting that individual molecular characterization of advanced phase CML patients may be increasingly necessary. It must be acknowledged that mutations of ASXL1 and other myeloid malignancy-associated genes have been detected at low levels by deep sequencing in both $\mathrm{Ph}$-negative and $\mathrm{Ph}$-positive clones of CML patients. These findings suggest that expression of $B C R-A B L 1$ may be insufficient for the development of CML which has implications for treatment with both TKIs and other novel agents $[18,30]$. Whether the ASXL1 mutation detected in this case was present in a $B C R$ - $A B L 1$-independent clone cannot be excluded or confirmed as NGS was not performed when the patient had achieved remission after chemotherapy; however, the high ASXL1 mutation VAF at presentation points to the ASXL1 mutation and BCR-ABL1 fusion coexisting in the same clone.

$\mathrm{BM}$ fibrosis, as evident in this case, is an infrequent but recurrent feature of CML. In the classical Philadelphia chromosome-negative myeloproliferative neoplasms, several lines of evidence link ASXL1 mutations with BM fibrosis: ASXL1 mutations are associated with a higher degree of BM fibrosis in primary myelofibrosis [31]; ASXL1 mutations are associated with an increased risk of myelofibrotic transformation in patients with essential thrombocythemia and polycythemia vera [32]; and ASXL1 mutations are more frequently detected in overt as opposed to prefibrotic primary myelofibrosis [33]. An association therefore potentially exists between ASXL1 mutations and BM fibrosis that requires further investigation in CML patients.

Despite the fact that there is no standardized approach to monitoring atypical $B C R-A B L 1$ transcript types, RT-qPCR has been adopted in some e6a2 BCR-ABL1 cases of CML correlating well with clinical course as in the case described herein. An emerging, alternative methodology to RT-qPCR is digital droplet PCR that allows for more accurate quantitation of target molecules without the use of standard curves. This approach has recently been applied to molecular monitoring of a CML patient with an e6a $2 B C R-A B L 1$ fusion, demonstrating an initial three-log reduction in transcripts with dasatinib monotherapy [34].

In conclusion, comprehensive molecular genetic characterization of the rare e6a2 $B C R-A B L 1$ fusion and accompanying mutations facilitates prospective molecular monitoring and may also provide targets for potential therapeutic intervention in CML patients with this hostile genotype. 


\section{Conflicts of Interest}

The authors declare that there are no conflicts of interest regarding the publication of this paper.

\section{References}

[1] S. E. Langabeer, "Is the BCR-ABL1 transcript type in chronic myeloid leukaemia relevant?" Medical Oncology, vol. 30, no. 2, article no. 508, 2013.

[2] A. Hochhaus, A. Reiter, H. Skladny et al., "A novel BCR-ABL fusion gene (e6a2) in a patient with Philadelphia chromosomenegative chronic myelogenous leukemia," Blood, vol. 88, no. 6 , pp. 2236-2240, 1996.

[3] M. Dupont, E. Jourdan, and J. Chiesa, "Identification of E6A2 BCR-ABL fusion in a Philadelphia-positive CML [1]," Leukemia, vol. 14, no. 11, pp. 2011-2012, 2000.

[4] B. Schultheis, L. Wang, R. E. Clark, and J. V. Melo, "BCR-ABL with an e6a2 fusion in a CML patient diagnosed in blast crisis [4]," Leukemia, vol. 17, no. 10, pp. 2054-2055, 2003.

[5] S. Colla, G. Sammarelli, S. Voltolini, M. Crugnola, P. Sebastio, and N. Giuliani, "E6a2 BCR-ABL transcript in chronic myeloid leukemia: is it associated with aggressive disease?" Haematologica, vol. 89, no. 5, pp. 611-613, 2004.

[6] K. A. Beel, J. Lemmens, H. Vranckx, J. Maertens, and P. Vandenberghe, "CML with e6a2 BCR-ABL1 transcript: An aggressive entity?” Annals of Hematology, vol. 90, no. 10, pp. 1241-1243, 2011.

[7] S. Hayette, I. Tigaud, X. Thomas et al., "Identification of a rare e6a2 BCR-ABL fusion gene during the disease progression of chronic myelomonocytic leukemia: A case report [4]," Leukemia, vol. 18, no. 10, pp. 1735-1736, 2004.

[8] M.-J. Grégoire, V. Latger-Cannard, A. Staal et al., "Identification of an acute basophilic leukaemia carrying a rare e6a2 BCRABL transcript," Acta Haematologica, vol. 116, no. 3, pp. 216-218, 2006.

[9] J. Yao, D. Douer, L. Wang, M. E. Arcila, K. Nafa, and A. Chiu, "A case of acute myeloid leukemia with e6a2 BCR$\mathrm{ABL}$ fusion transcript acquired after progressing from chronic myelomonocytic leukemia," Leukemia Research Reports, vol. 7, pp. 17-19, 2017.

[10] C. Popovici, S. Cailleres, M. David, M. Lafage-Pochitaloff, D. Sainty, and M.-J. Mozziconacci, "E6a2 BCR-ABL fusion with BCR exon 5-deleted transcript in a Philadelphia positive CML responsive to imatinib," Leukemia \& Lymphoma, vol. 46, no. 9, pp. 1375-1377, 2005.

[11] M. Breccia, L. Cannella, D. Diverio et al., "Isolated thrombocytosis as first sign of chronic myeloid leukemia with e6a2 $\mathrm{BCR} / \mathrm{ABL}$ fusion transcript, JAK2 negativity and complete response to imatinib," Leukemia Research, vol. 32, no. 1, pp. 177180, 2008.

[12] S. Schnittger, U. Bacher, W. Kern, T. Haferlach, B. Hertenstein, and C. Haferlach, "A new case with rare e6a2 BCR-ABL fusion transcript developing two new resistance mutations during imatinib mesylate, which were replaced by T315I after subsequent dasatinib treatment," Leukemia, vol. 22 , no. 4, pp. 856858, 2008.

[13] H. K. Vefring, F. X. E. Gruber, L. Wee et al., "Chronic myelogenous leukemia with the e6a2 bcr-abl and lacking imatinib response: Presentation of two cases," Acta Haematologica, vol. 122, no. 1, pp. 11-16, 2009.
[14] S. Corm, A. Renneville, E. Rad-Quesnel, N. Grardel, C. Preudhomme, and B. Quesnel, "Successful treatment of imatinibresistant acute megakaryoblastic leukemia with e6a2 BCR/ABL: Use of dasatinib and reduced-conditioning stem-cell transplantation [10]," Leukemia, vol. 21, no. 11, pp. 2376-2377, 2007.

[15] S. E. Langabeer, M. Crampe, J. Kelly, K. Fadalla, G. Connaghan, and E. Conneally, "Nilotinib and allogeneic stem cell transplantation in a chronic myeloid leukemia patient with e6a2 and ela2 BCR-ABL transcripts," Leukemia Research, vol. 34, no. 8, pp. e204-e205, 2010.

[16] P. Rohon, M. Divoka, L. Calabkova et al., "Identification of e6a2 BCR-ABL fusion in a philadelphia-positive CML with marked basophilia: Implications for treatment strategy," Biomedical Papers, vol. 155, no. 2, pp. 187-190, 2011.

[17] V. Grossmann, A. Kohlmann, and M. Zenger, "A deep-sequencing study of chronic myeloid leukemia patients in blast crisis (BC-CML) detects mutations in $76.9 \%$ of cases," Leukemia, vol. 25, no. 3, pp. 557-560, 2011.

[18] M. Schmidt, J. Rinke, V. Schäfer et al., "Molecular-defined clonal evolution in patients with chronic myeloid leukemia independent of the BCR-ABL status," Leukemia, vol. 28, no. 12, pp. 2292-2299, 2014.

[19] C. Lai, J. E. Karp, and C. S. Hourigan, "Precision medicine for acute myeloid leukemia," Expert Review of Hematology, vol. 9, no. 1, pp. 1-3, 2016.

[20] M. Baccarani, M. W. Deininger, G. Rosti et al., "European LeukemiaNet recommendations for the management of chronic myeloid leukemia: 2013," Blood, vol. 122, no. 6, pp. 872-874, 2013.

[21] S. O’Brien, J. P. Radich, C. N. Abboud et al., "Chronic myelogenous leukemia, version 1.2015," Journal of the National Comprehensive Cancer Network, vol. 12, no. 11, pp. 1590-1610, 2014.

[22] J. Gabert, E. Beillard, V. H. J. van der Velden et al., "Standardization and quality control studies of 'real time' quantitative reverse transcriptase polymerase chain reaction of fusion gene transcripts for residual disease detection in leukemia-A Europe Against Cancer Program," Leukemia, vol. 17, no. 12, pp. 23182357, 2003.

[23] J. J. M. Van Dongen, E. A. Macintyre, J. A. Gabert et al., "Standardized RT-PCR analysis of fusion gene transcripts from chromosome aberrations in acute leukemia for detection of minimal residual disease. Report of the BIOMED-1 concerted action: investigation of minimal residual disease in acute leukemia," Leukemia, vol. 13, no. 12, pp. 1901-1928, 1999.

[24] K. Haslam, M. A. Catherwood, E. Dobbin, A. Sproul, S. E. Langabeer, and K. I. Mills, "Inter-Laboratory Evaluation of a Next-Generation Sequencing Panel for Acute Myeloid Leukemia," Molecular Diagnosis \& Therapy, vol. 20, no. 5, pp. 457-461, 2016.

[25] A. Pellagatti and J. Boultwood, "The molecular pathogenesis of the myelodysplastic syndromes," European Journal of Haematology, vol. 95, no. 1, pp. 3-15, 2015.

[26] J. Boultwood, J. Perry, R. Zaman et al., "High-density single nucleotide polymorphism array analysis and ASXL1 gene mutation screening in chronic myeloid leukemia during disease progression," Leukemia, vol. 24, no. 6, pp. 1139-1145, 2010.

[27] H. Makishima, A. M. Jankowska, M. A. McDevitt et al., "CBL, CBLB, TET2, ASXL1, and IDH1/2 mutations and additional chromosomal aberrations constitute molecular events in chronic myelogenous leukemia," Blood, vol. 117, no. 21, pp. e198e206, 2011. 
[28] E. Togasaki, J. Takeda, K. Yoshida et al., "Frequent somatic mutations in epigenetic regulators in newly diagnosed chronic myeloid leukemia," Blood Cancer Journal, vol. 7, no. 4, p. e559, 2017.

[29] M. F. Zahid, M. M. Patnaik, N. Gangat, S. K. Hashmi, and D. A. Rizzieri, "Insight into the molecular pathophysiology of myelodysplastic syndromes: targets for novel therapy," European Journal of Haematology, vol. 97, no. 4, pp. 313-320, 2016.

[30] T. Kim, M. S. Tyndel, H. J. Kim et al., "Spectrum of somatic mutation dynamics in chronic myeloid leukemia following tyrosine kinase inhibitor therapy," Blood, vol. 129, no. 1, pp. 3847, 2017.

[31] P. Guglielmelli, G. Rotunno, A. Pacilli et al., "Prognostic impact of bone marrow fibrosis in primary myelofibrosis. A study of the AGIMM group on 490 patients," American Journal of Hematology, vol. 91, no. 9, pp. 918-922, 2016.

[32] B. L. Stein, D. M. Williams, C. O'Keefe et al., "Disruption of the ASXL1 gene is frequent in primary, post-essential thrombocytosis and post-polycythemia vera myelofibrosis, but not essential thrombocytosis or polycythemia vera: Analysis of molecular genetics and clinical phenotypes," Haematologica, vol. 96, no. 10, pp. 1462-1469, 2011.

[33] P. Guglielmelli, A. Pacilli, G. Rotunno et al., "Presentation and outcome of patients with 2016 WHO diagnosis of prefibrotic and overt myelofibrosis," Blood, vol. 129, no. 24, pp. 3227-3236, 2017.

[34] A. Zagaria, L. Anelli, N. Coccaro et al., " BCR-ABL1 e6a2 transcript in chronic myeloid leukemia: biological features and molecular monitoring by droplet digital PCR," Virchows Archiv, vol. 467, no. 3, pp. 357-363, 2015. 


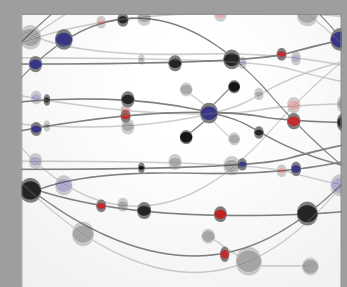

The Scientific World Journal
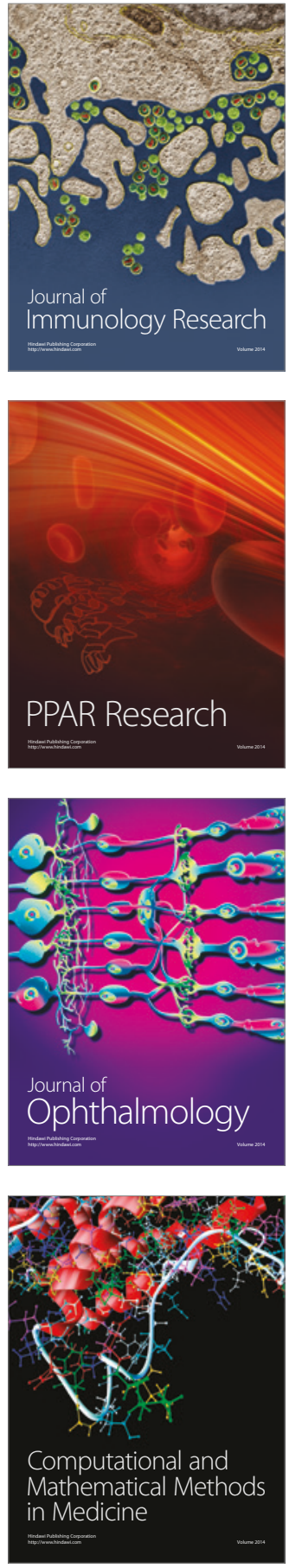

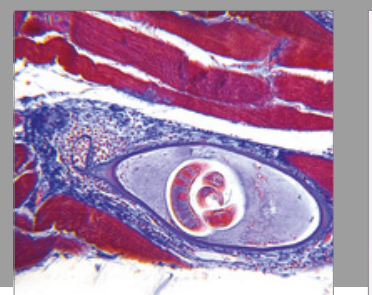

Gastroenterology Research and Practice
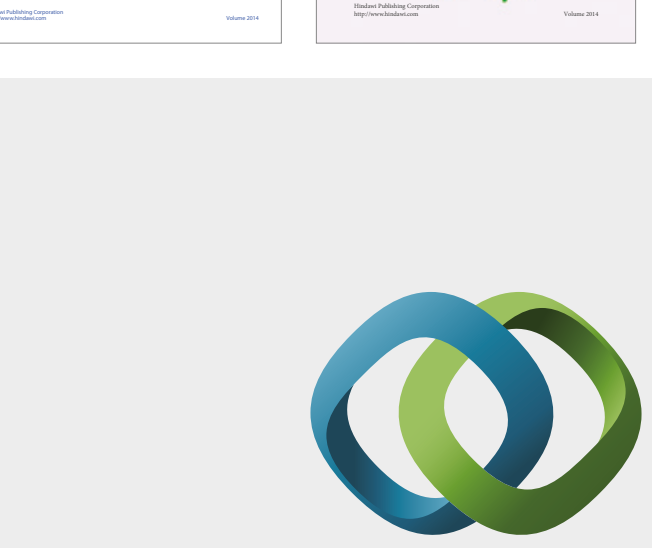

\section{Hindawi}

Submit your manuscripts at

https://www.hindawi.com
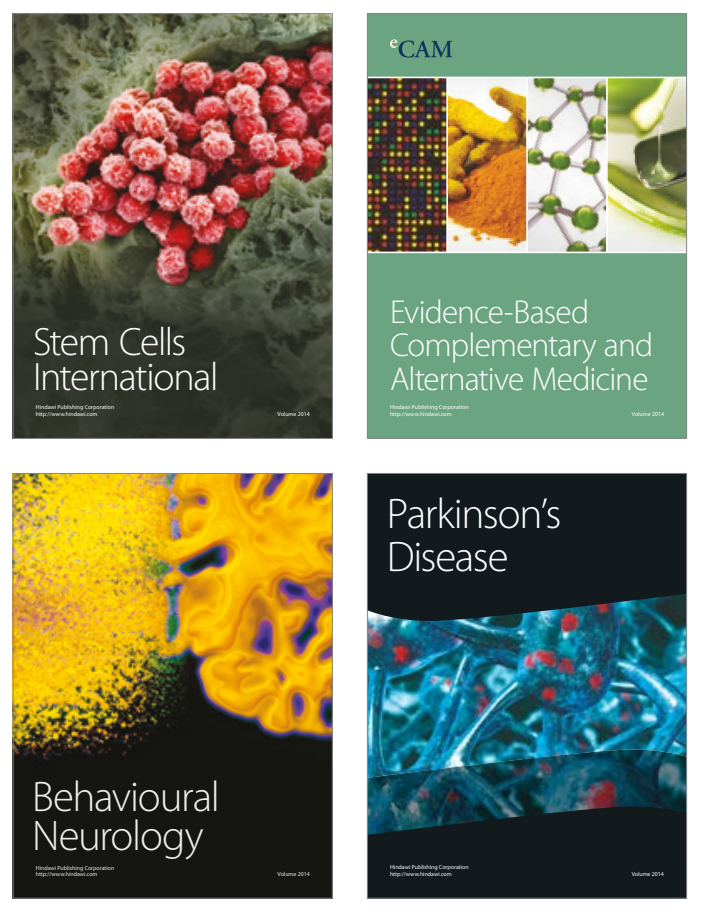
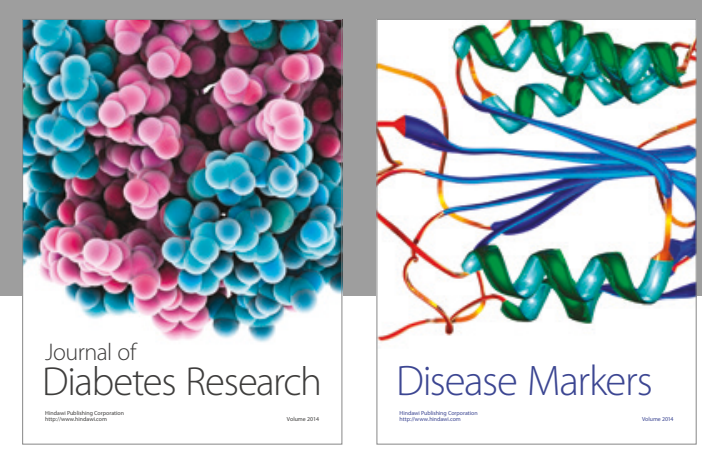

Disease Markers
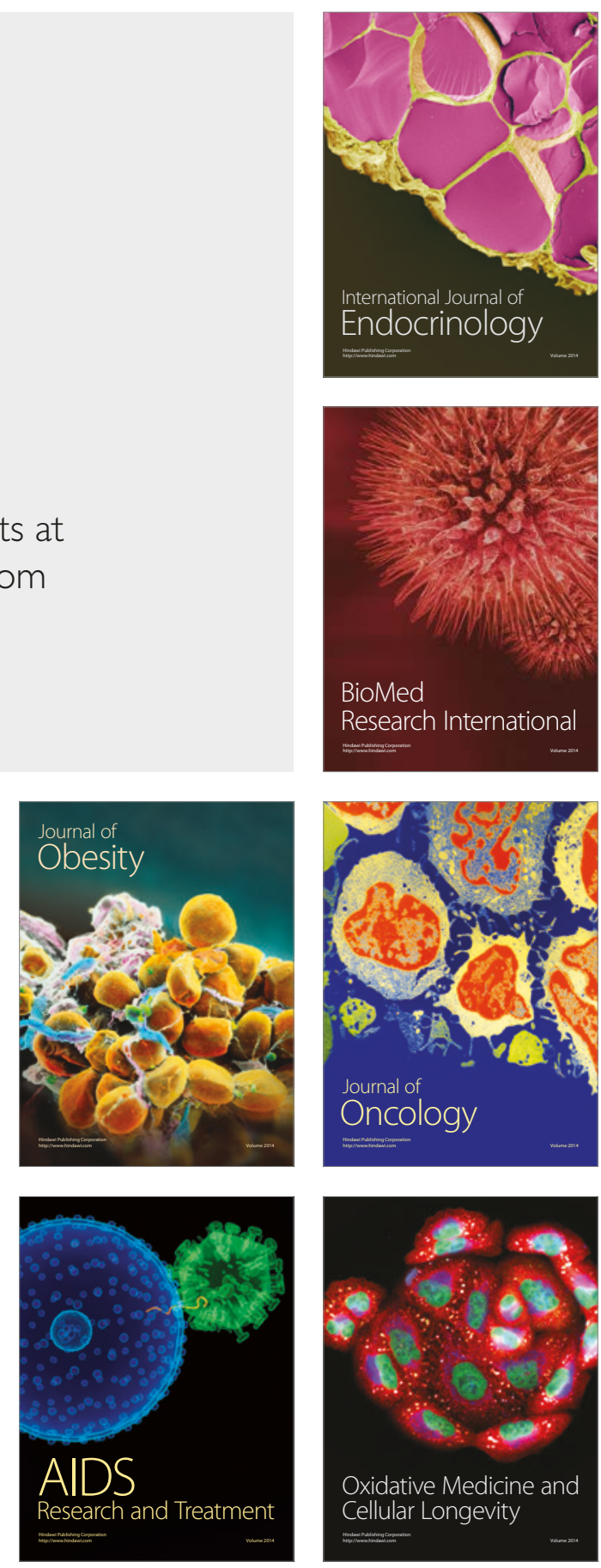